\title{
Overview of the 3rd DECOR Workshop
}

\author{
Frederic Andres \\ Digital Content and Media Sciences Research Division \\ National Institute of Informatics \\ Tokyo, Japan \\ 0000-0002-5003-7579
}

\author{
William Grosky \\ College of Engineering and Computer Science \\ University of Michigan-Dearborn \\ Dearborn, USA \\ wgrosky@umich.edu
}

\author{
Gheorghita Ghinea \\ Department of Computer Science \\ Brunel University London \\ London, UK \\ 0000-0003-2578-5580
}

\author{
Maria C.A. Leite \\ Department of Mathematics and Statistics \\ University of South Florida St. Petersburg \\ St. Petersburg, USA \\ 0000-0002-8096-7152
}

\begin{abstract}
This extended abstract introduces the 3rd International Workshop on Data Engineering Meets Intelligent Food Cooking Recipes 2020 in conjunction with the 36th IEEE International Conference on Data Engineering 2020 (ICDE). The objectives of this workshop were to review the current development and challenges at the crossroad of data engineering and Intelligent Food \& Cooking Recipes.

Index Terms-DECOR, Data Engineering, Intelligent Food, Cooking Recipes
\end{abstract}

\section{Motivations and Objectives}

The past decade has seen an enormous growth in the research related to the digital food and cooking recipes underpinnings of data engineering, as food is essential for human life and related health. The ability to collect, store, process, and evaluate cooking recipes has advanced immeasurably, and data science-driven methods have had unprecedented impact on food experience sharing and recommendations at large, mainly because of their success in analysing and predicting human cooking expectation, flavour, and taste preferences. Data Engineering stands to benefit from the food computing and recipe cooking revolution in similar ways, but realizing this vision requires thoughtful and concerted effort. The Third Workshop on Data Engineering meets Intelligent Food and COoking Recipes (DECOR) aims to accelerate research in data science by providing a forum for the latest innovations in the intersection of Data Engineering and Intelligent Food and Cooking Recipes. This workshop is specifically focused on data science innovations that accelerate the organization, integration, access, and sharing of digital objects in support of the Intelligent Food and Cooking Recipes domain. This domain comprises not only the process of cooking, but also includes intelligent methods for enhancing human-food interactions, ranging from devising technology, playful interactions, multisensory experience design, understanding crosscultural food eating habits and perception, as well as food choices and thirs health connections. Consequently, increasing the ability of influencing food eating habits and choices that promote, simultaneously, healthful eating-decisions and creative new human-food interaction experiences. According to Trends on the Gartner Hype Cycle for Artificial Intelligence, Data Engineering will contribute to solve the complexity of a human-centred partnership model of people and AI working together to enhance cognitive performance related to sharing dish tasting experiences. Resilience and real-time adaptability are essential for health monitoring and predictive maintenance of complex food menus and diets where data-driven decisions can improve health and dish experience quality. The research design itself will enable us to integrate the user requirements and expectations, where data-driven modelling can accelerate the design cycle while yielding more capable and predictable social media tools for food experience and recommendation sharing.

\section{TOPICS AND SCHEDULE}

The acceptance rate for DECOR 2020 was 56\%. The program is as follows:

\section{A. Keynotes Speakers}

a) Prof. Alan Hevner: took the stage on Monday on Monday morning from 9:15 AM to 10:00 AM as the opening keynote. He spoke about how Design Science Research can benefit Food Science and Data Engineering. He explored both designed artifacts and evidence of their impacts, along with a fuller scientific understanding via design theories of why the artifacts provide enhancements (or, disruptions) to the relevant application contexts.

b) Prof. Pascal Hitzler: took the stage on Monday afternoon from 1:30 PM to 2:15 PM. His keynote focused on Knowledge Graphs applied to the domain of cooking recipes. This enabling modeling tool is currently being established as a major paradigm for data sharing, integration and reuse. $\mathrm{He}$ 
discussed the current state-of-the-art, and presented recent and ongoing work on the design of reusable knowledge graphs, based on modular ontology modeling.

\section{B. Paper Session 1 10:30 AM to 12:00 PM}

Recipe modeling and ingredient classification are still a challenging domain. In the morning session original research papers in this arena were presented:

- A Named Entity Based Approach to Model Recipes, presented by Devansh Batra from Department of Information Technology NSUT, New Delhi. The research presented focus on a structural model that can accurately represent the recipe as well as on a pipeline to infer the best representation of the recipe. Batra discussed how this mined information model can have several applications which include translating recipes between languages, determining similarity between recipes, generation of novel recipes and estimation of the nutritional profile of recipes.

- Nutritional Profile Estimation in Cooking Recipes presented by Jushaan Kalra from Department of Computer Engineering Delhi Technological University, India. Kalra introduced a scalable method for nutritional profile estimation of recipes from their ingredients section. The availability of an accurate nutrition profile of recipes is an important feature for food databases with several applications including nutritional assistance, recommendation systems, and dietary analytics. However, often online databases have incomplete and unreliable set of nutritional details. Kalra discussed how the proposed innovative protocol is robust, compatible with any nutritional database, easily replicable and solves one of the foremost problems with dietary analysis and food recommendation systems.

- Food Recipe Alternation and Generation with Natural Language Processing Techniques presented by Qiangwen $\mathrm{Xu}$ from Fordham University, USA. Xu presented his paper that focus on how to apply natural language processing techniques such as word embedding to assist people to choose alternative ingredients or recipes, a quite challenging task. He also discussed how they build language model-N-gram and neuronal model capable of creating new recipes preserving authenticity of flavor of certain cuisine.

\section{Paper Session 2: 2:15PM to 3:00 PM}

A regular paper and a short paper focused on similarities and differences between cuisines:

- Hierarchical Clustering of World Cuisines presented by Tript Sharma, Delhi Technological University, India. The presenter discussed two proposed methodologies for fingerprinting cuisines and identifying their interdependence. The underlying hypothesis in their work is that some patterns, which are common across a subset of cuisines would be found, which defines the cuisines 'similarity/closeness' with each other. The work shows how cooking practices and methods are shared by neighbouring regions. This analysis is important from a historical and cultural point of view as it helps in appreciating how cooking practices are distributed across the world. Furthermore, it also provide a verbose list of patterns identified in the cuisines such as compound pattern, combination of ingredients, processes and utensils.

- (Short Paper) Classification of Cuisines from Sequentially Structured Recipes presented by Utkarsh Upadhyay, Jamia Millia Islamia University, India. Upadhyay's talk focus on a novel approach to characterize cuisines based on their substructures such as ingredients, cooking processes and sequences as well as utensils. A complex fusion of these substructures intrinsic to a region defines the identity of a cuisine. Previous cuisine classification use unstructured recipes without accounting for details of cooking techniques. In reality, the cooking processes/techniques and their order are highly significant for the recipe's structure and hence for its classification.

\section{Panel discussion session - Current Challenges and Future} Directions: 3:30 PM to 4:30 PM

Questions posed to the panel discussion session participants included the following:

- Describe a scenario using tools of the future in the realworld and give your ideas of how your wish list of tools could be used on a day-to-day basis.

- How could sensor-based taste data be integrated into an overall useful tool?

- What other sorts of data, besides recipes, would be useful to examine?

- How could the notion of a temporal sequence be used into our overall approach, and what is the enabling tool-base for this area of study?

- What are some open questions that you believe are important to address in your work, and, in general, for the field, bridging Intelligent Food and Data Engineering?

- Share with us your ideas on developing food tasting and personal experience privacy-by-design, as well as food security, as sub-fields of the integration of Data Engineering and Intelligent Food.

- What actions/strategies would be helpful to implement in order to attract students and researchers to this field?

\section{E. Best Paper Award}

This year the recipient of the award is Qiangwen Xu, Fordham University, USA, with the paper titled "Food Recipe Alternation and Generation with Natural Language Processing Techniques."

\section{ACKNOWLEDGMENT}

We would like to express our appreciation to NII, Brunel University London, University of South Florida St. Petersburg, and University of Michigan-Dearborn for their support of the DECOR workshop. We would like to warmly thank Damien, Gabriel, Hien and Mustafa for their immense support during the DECOR 2020 workshop. 


\section{REFERENCES}

[1] W. Min, S. Jiang, L. Liu, Y. Rui, and R. Jain. 2019. A Survey on Food Computing. ACM Comput. Surv. 1, 1.

[2] W. Chang. 2019. Applying ANP to analyse sensory experience in restaurants, British Food Journal, Vol. 122 No. 1, pp. 122-135. https://doi.org/10.1108/BFJ-07-2019-0497

[3] J. Hillen. 2019. Web scraping for food price research, British Food Journal, Vol. 121 No. 12, pp. 3350-3361. https://doi.org/10.1108/BFJ02-2019-0081

\section{CO-CHAIR BIOGRAPHY}

- Dr. Hdr. Frederic Andres received his Ph.D. in Information Systems from the University of Paris VI "Pierre et Marie Curie" in 1993 and his Dr. Habilitation in Informatics from the University of Nantes in 2000. Dr. Frederic Andres has been associate professor in the Digital Content and Media Sciences Research Division of the National Institute of Informatics (NII) in Tokyo, Japan since 2000 and of Sokendai (Tokyo) since 2002. $\mathrm{He}$ is author of more than 150 papers on international journals, books and conferences. His main research areas of interest include several topics such as collective intelligence-based community management, early warning management, pedagogy and didactical web-based science education for all, intelligent cooking recipe and water crystal computing. He has been co-organising the International DECOR workshop in conjunction of ICDE 2018 (Paris, April 2018) and ICDE 2019 (Macau, April 2019). His team won the 1st prize in IEEE Brain Data Bank Challenges and Competitions on COMPSAC 2018, July 2018.

- William I. Grosky is currently a professor in the Department of Computer and Information Science at the University of Michigan-Dearborn. Previously, he was chair of the department for 16 years. Before joining UMD in 2001, he was professor and chair of the Department of Computer Science at Wayne State University, as well as an assistant professor of Information and Computer Science at the Georgia Institute of Technology in Atlanta. His current research interests are in data science, multimedia information systems, text and image mining, and natural language processing. Grosky received his B.S. in mathematics from MIT in 1965, his M.S. in applied mathematics from Brown University in 1968, and his Ph.D. from Yale University in 1971. He has given many short courses in the area of database management for local industries and has been invited to lecture on multimedia information systems world-wide. Serving also on many database and multimedia conference program committees, he was an Editor-in-Chief of IEEE Multimedia, and is currently on the editorial boards of over 20 journals. He has also been the main advisor for 24 Ph.D. recipients.

- Dr. Gheorghita (George) Ghinea is a Professor in Mulsemedia Computing in the Department of Computer Science, at Brunel University. Dr. Ghinea's research activities lie at the confluence of Computer Science, Media and Psychology. In particular, his work focuses on the area of perceptual multimedia quality and how one builds end-to-end communication systems incorporating user perceptual requirements. To this end, he has proposed the concept of mulsemedia - multiple sensorial media - a concept which goes beyond traditional multimedia, integrating media which that go beyond the audiovisual, and engage more than three of human senses. Dr. Ghinea has applied his expertise in areas such as eye-tracking,telemedicine, multi-modal interaction, and ubiquitous and mobile computing, leading a team of 8 researchers in these areas. He has over 300 publications in his research field and was the lead Brunel investigator of a H2020 project NEWTON (http://www.newtonproject.eu/) applying mulsemedia to STEM learning across Europe.

- Maria C.A. Leite. Associate professor, Department of Mathematics Statistics at University of South Florida St. Petersburg. A/Prof Leite research emphasizes the applied and theoretical study of nonlinear dynamics of coupled networks. The aim is to advance the understanding of the behavior of complex systems. One of her currents interests is the application of techniques such as mathematical modeling, control theory, and data sciences to gain insights into Intelligent Food domain. She has been co-organising the International DECOR workshop since 2018 in conjunction of ICDE 2018 (Paris, April 2018) and ICDE 2019 (Macau, April 2019). 